\title{
Extraction of S-matrix pole structure using deep learning
}

\section{Denny Lane B. Sombillo ${ }^{*}$ *}

National Institute of Physics, University of the Philippines Diliman, Quezon City 1101, Philippines Research Center for Nuclear Physics (RCNP), Osaka University, Ibaraki, Osaka 567-0047, Japan

E-mail: dbsombillo@up.edu.ph, sombillo@rcnp.osaka-u.ac.jp

In this paper, we demonstrate how deep learning can be used as a unified model-selection tool in the analysis of hadron-hadron scattering. We consider the problem of finding the number of poles in each unphysical Riemann sheet to reproduce the elastic $\pi N$ amplitude. The model space is constructed using 35 pole-based models with a maximum of 4 poles distributed in each Riemann sheet. The uncertainty due to the limited energy resolution is included in the generation of training amplitudes. The learning of the deep neural network is initiated using the curriculum technique, achieving a final training and testing accuracies of $76.5 \%$ and $80.4 \%$, respectively. Due to the presence of error bars on the amplitude, it is expected that the experimental data can be described by more than one model. Thus, to realize the multiple descriptions of a given experimental data in our analysis, we utilize the error bars and generate $10^{6}$ inference amplitudes to be fed directly to the trained neural network. Out of the 35 models, only 4 models are identified by the trained deep neural network to describe the $\pi N$ scattering data. The most favored pole structure for the $\pi N$ amplitude is one pole in each nearby sheet and two poles in the remote sheet. Further numerical analyses show that the deep learning framework is also robust and does not depend on how the inference amplitudes are generated from the experimental data.

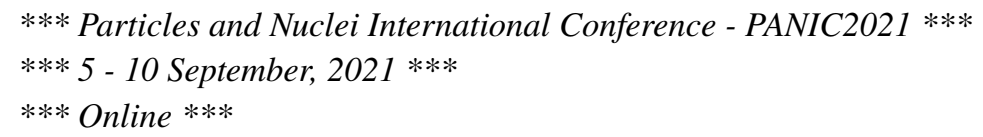

${ }^{*}$ Speaker 


\section{Introduction}

There is a growing interest in the exploration of hadron spectroscopy due to the abundance of recently discovered near-threshold phenomena [1-3]. Understanding the origin of the observed peak structures is crucial to unravel the mechanism of color confinement. The conventional approach in the analysis is to utilize a dynamical model with parameters fitted to the data and then interpret the experimental results. The model-fitting scheme is useful if we want to constrain or estimate the parameters of the chosen model but it is not optimal when we want to do model selection. It turns out that deep learning can be used as a unified model-selection framework in the analysis of experimental data.

Deep learning is becoming a common experimental tool in particle-physics analysis, with applications ranging from event selection to particle identification [4, 5]. Recently, we made a series of steps to apply deep learning in amplitude analysis [6,7]. We trained a set of deep neural network models to pick up the subtle differences between a bound and a virtual enhancements given only the partial cross-sections with near-threshold pole. The neural networks were trained to utilize the unbalanced distribution of S-matrix singularities in the single channel scattering and successfully identified the s-wave cross-section with bound state enhancement. In the present work, we demonstrate how deep learning can be used as a unified model-selection framework to analyze the experimental data.

\section{Pole-based models, neural network design and training}

The nature of peak structures in the scattering data can be inferred by invoking the pole-counting argument of Morgan and Pennington [8-10]. That is, we can deduce the nature of resonance by knowing the number of poles in each Riemann sheet. In most cases, a given experimental data requires more than one pole for complete description (see for example Refs. [11, 12]). Thus, each pole structure can be considered as a model describing the nature of resonance. In this study, we consider the $\pi N$ s-wave elastic amplitude shown in Fig.1(a) as our experimental data and utilize deep learning to extract the pole-structure model.

The first step in deep learning approach is to construct the training dataset containing input amplitudes with pole-based model as output labels. Applying analyticity, unitarity, and Hermiticity requirements, we can write the scattering amplitude as

$$
T_{1,1}\left(p_{1}, p_{2}\right)=\frac{1}{2 i}\left(\prod_{n=1}^{N} \frac{D_{n}\left(-p_{1}, p_{2}\right)}{D_{n}\left(p_{1}, p_{2}\right)}-1\right)
$$

where the subscript 1 corresponds to the lower $\pi N$ channel and 2 to the higher $\eta N$ channel. Each $D_{n}\left(p_{1}, p_{2}\right)$ is a Jost function designed to produce exactly one pole near the scattering region on the desired unphysical sheet. A large size of input training amplitudes is generated by randomly placing the poles on any position in each Riemann sheet. An output label is assigned to each amplitude based on the number of Riemann sheet poles used. We also use distant poles to simulate the background singularities of the S-matrix. Note that all the poles used to generate one amplitude are independent of each other, which means that no dynamical model is favored in our formulation. For practical reasons, we only consider pole-based models having no more than $N=4$ poles. 


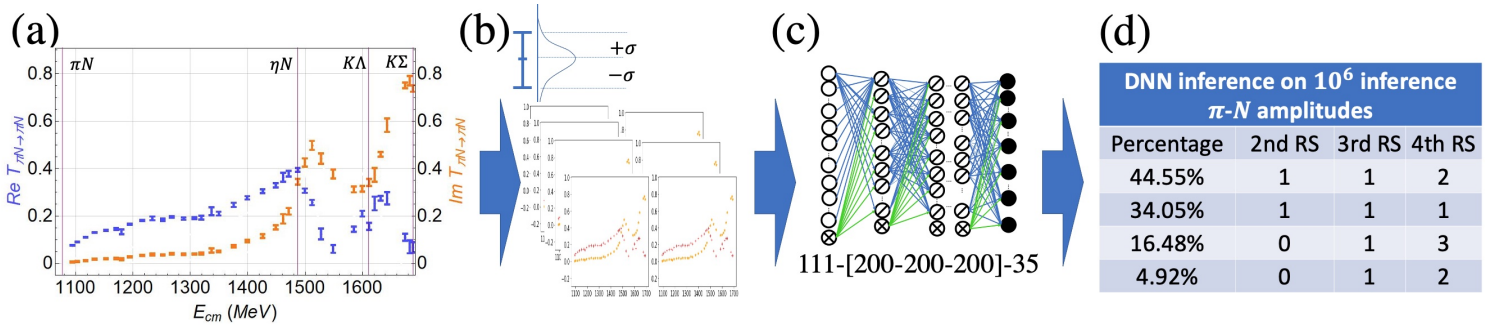

Figure 1: Post-training inference analysis. The s-wave $\pi N$ scattering GW-SAID data in [13] is used as the experimental data (a). In (b), a total of $10^{6}$ inference amplitudes are generated using the error bars in (a). The inference amplitudes are directly fed to the trained neural net (c) giving the final inference result in (d).

This restriction gives us 35 different models ranging from no nearby poles up to one pole in each unphysical sheet.

Initially, we experiment with different architectures and find that the design shown in Fig.1(c) is optimal to our model-selection task [14]. Our chosen deep neural network has 111 nodes (open circles) to take the random energy points, the real and, the imaginary parts of the amplitudes at the input layer. We use three hidden layers with 200 nodes in each layer (slashed circles) equipped with ReLU activation function. Finally, we have 35 output nodes representing the 35 pole-based models, each with softmax activation function. All layers except the output layer has an extra node for the bias (crossed circles).

The next step is to train the chosen deep neural network. Due to the inherent noise exhibited by the random input energy points and random background singularities in each training amplitude, the learning process becomes difficult. We employ the curriculum technique where the training proceeds by presenting one model at time. Further details can be seen in Ref.[14]. The technique successfully initiated the learning, giving us a final training and testing accuracies of $76.5 \%$ and $80.4 \%$, respectively. The trained neural network can now be deployed to analyze the experimental data.

\section{Inference results}

Prior to the deep learning analysis we anticipate that, due to the experimental uncertainty, the data can be described by more than one model. We can accommodate the multiple description of the experimental data by using the error bars in the inference stage of the analysis. In Fig.1(b), we generate $10^{6}$ inference amplitudes by drawing points on each error bar using a Gaussian distribution. Then, we feed all of the inference amplitude to the trained neural network and count the number of outcomes. The inference result is shown in Fig.1(d). Note that out of the 35 models, only 4 models are assigned to the experimental data. We get the highest count for the the 4-pole model with 1 pole in the second sheet, one pole in the third and two poles in the fourth. The three outcomes with smaller counts reflect the multiple interpretation of the data due to the error bar.

It can also be shown that the inference result is independent of the chosen probability distribution used to generate the inference amplitudes. One can use the uniform distribution and obtain similar outcomes shown in Fig.1(d) as demonstrated in [14]. Thus, our proposed deep learning modelselection framework provides a robust analysis of the experimental data. 


\section{Conclusion and Outlook}

We show how deep learning can be used as a unified model-selection framework covering a wider model space. In some sense, the proposed method automates the model-fitting scheme where different models are compared and evaluated based on the goodness of fit. Our method eliminates the subjective comparison of models exhibiting almost the same goodness of fit. For future study, we intend to use the deep learning technique that can also extract the pole parameters to give an unambiguous and model-independent description of the experimental data.

\section{Acknowledgements}

I would like to thank the many fruitful discussions with Prof. Atsushi Hosaka, Dr. Toru Sato and Dr. Yoichi Ikeda. Special thanks to Dr. Noriyoshi Ishii for setting up the computing facilities needed to perform the deep learning analysis. I am also grateful to the generosity of the Research Center of Nuclear Physics (RCNP), Osaka University for hosting my almost three-year stay in Japan. Finally, the author acknowledges the support of DOST-SEI ASTHRDP through its postdoctoral research fellowship.

\section{References}

[1] S. L., Olsen, T. Skwarnicki, and D. Zieminska, Nonstandard heavy mesons and baryons: Experimental evidence, Rev. Mod. Phys. 90, 015003 (2018)

[2] F.-K. Guo, C. Hanhart, U.-G. Meißner, Q. Wang, Q. Zhao, and B.-S. Zou, Rev. Mod. Phys. 90, 015004 (2018)

[3] B.-S. Zhou, Sci. Bull. 66, 1258 (2021)

[4] G. Carleo, I. Cirac, K. Cranmer, L. Daudet, M. Schuld, N. Tishby, L. Vogt-Maranto, and L. Zdeborová, Rev. Mod. Phys. 91, 045002 (2019)

[5] D. Bourilkov, Int. J. Mod. Phys. A. 34, 1930019 (2020)

[6] D. L. B. Sombillo, Y. Ikeda, T. Sato, and A. Hosaka, Phys. Rev. D. 102, 016024 (2020)

[7] D. L. B. Sombillo, Y. Ikeda, T. Sato, and A. Hosaka, Few-Body Syst. 62, 52 (2021)

[8] D. Morgan, Nucl. Phys. A 543, 632 (1993)

[9] D. Morgan, and M. R. Pennington Phys. Rev. D 48, 1185 (1993)

[10] D. Morgan, and M. R. Pennington Phys. Lett. B 258, 444 (1991)

[11] Z.-Y. Wang, H. A. Ahmed, and C. W. Xiao Eur. Phys. J. C 81, 833 (2021)

[12] V. K. Magas, E. Oset, and A. Ramos hys. Rev. Lett. 95, 052301 (2005)

[13] https://gwdac.phys.gwu.edu/analysis/pin_analysis.html

[14] D. L. B. Sombillo, Y. Ikeda, T. Sato, and A. Hosaka, Phys. Rev. D 104, 3 (2021) 